\title{
RX J0944.5+0357: A Probable Intermediate Polar*
}

\author{
Patrick A. Woudt and Brian Warner \\ Dept. of Astronomy, University of Cape Town, Rondebosch 7700, South Africa \\ E-mail: pwoudt@circinus.ast.uct.ac.za,warner@physci.uct.ac.za
}

30 October 2002

\begin{abstract}
From optical photometry the cataclysmic variable RX J0944.5+0357 is shown to have a double-peaked pulse profile with a period $\sim 2160 \mathrm{~s}$. The two peaks vary rapidly in relative amplitude. Often most of the optical power is concentrated in the first harmonic of the $2160 \mathrm{~s}$ modulation; RX J0944.5+0357 therefore probably belongs to the relatively rare class of two-pole accreting intermediate polars exemplified by YY Dra and V405 Aur.
\end{abstract}

Keywords: dwarf novae, binary stars, cataclysmic variables

\section{Introduction}

Many of the X-Ray sources in the ROSAT All-Sky Survey have been identified optically in the Hamburg objective prism survey (Hagen et al. 1995), among which are several cataclysmic variables (CVs) (Jiang et al. 2000). The source RX J0944.5+0357 (= 1RXS J094432.1+035738; hereafter RXJ0944), in the constellation Sextans, was observed spectroscopically by Jiang et al. and found to have H I and He I emission lines typical of a CV. Further spectroscopic study by Mennickent et al. (2002) showed the presence of absorption bands in the red, characteristic of a secondary with a spectral type near M2. Observations by the VSNET group have identified two dwarf nova-like outbursts, in January and June 2001, during which RXJ0944 rose to V 13 from its quiescent magnitude of $\mathrm{V} \sim 16.2$. Mennickent et al. confirmed the spectroscopically determined orbital period $\left(P_{\text {orb }}\right)$ of $0.1492 \mathrm{~d}(3.581$ h) reported to them by Thorstensen \& Fenton. Mennickent et al. also provided the first high speed photometry of RXJ0944 in which large amplitude variations $(\sim 0.5 \mathrm{mag})$ were found on time scales of $10 \mathrm{~min}$ to $2 \mathrm{~h}$. They did not report any coherent signals in their photometry.

\footnotetext{
* This paper uses observations made from the South African Astronomical Observatory (SAAO).
}

(c) 2019 Kluwer Academic Publishers. Printed in the Netherlands. 
Table I. Observing log.

\begin{tabular}{ccccccc}
\hline Run No. & $\begin{array}{c}\text { Date of obs. } \\
\text { (start of night) }\end{array}$ & $\begin{array}{c}\text { HJD of first obs. } \\
(+2452000.0)\end{array}$ & $\begin{array}{c}\text { Length } \\
(\mathrm{h})\end{array}$ & $\begin{array}{c}t_{\text {in }} \\
(\mathrm{s})\end{array}$ & Tel. & $\begin{array}{c}<\mathrm{V}> \\
(\mathrm{mag})\end{array}$ \\
\hline S6324 & 22 March 2002 & 356.25290 & 4.98 & 15 & 40 -in & 16.4 \\
S6331 & 23 March 2002 & 357.40201 & 1.47 & 15 & 40 -in & 16.5 \\
S6340 & 25 March 2002 & 359.28466 & 0.89 & 30 & 40 -in & 16.5 \\
S6341 & 02 April 2002 & 367.23471 & 4.96 & 6 & 74 -in & 16.6 : \\
S6350 & 06 April 2002 & 371.23004 & 1.28 & 8 & 74 -in & $16.5:$ \\
S6362 & 09 April 2002 & 374.22997 & 3.05 & 20 & 40 -in & 16.5 \\
S6366 & 10 April 2002 & 375.28856 & 2.46 & 20 & 40 -in & 16.3 \\
S6370 & 11 April 2002 & 376.22836 & 3.26 & 20 & 40 -in & 16.5 \\
S6386 & 14 April 2002 & 379.23542 & 4.09 & 45 & 40 -in & 16.5 \\
S6391 & 15 April 2002 & 380.25135 & 3.36 & 10 & 40 -in & 16.5 \\
\hline
\end{tabular}

Notes: '?' denotes an uncertain value, $t_{i n}$ is the integration time.

\section{Photometric Observations}

We have used the University of Cape Town CCD Photometer (O'Donoghue 1995), attached to the 74-in and 40-in telescopes at the Sutherland site of the South African Astronomical Observatory, to observe RXJ0944 at time resolutions down to 6 s. Table 1 gives the log of our photometric observations and Figure 1 shows the resulting light curves.

A Fourier Transform (FT) of the entire data set shows no power at the spectroscopic period or its first harmonic, so we deduce that RXJ0944 is of quite low inclination. From the radial velocity amplitude of $75 \mathrm{~km} \mathrm{~s}^{-1}$ Mennickent et al. reasoned that the inclination probably lies in the range $30^{\circ}-60^{\circ}$; our result indicates that it is probably at the lower end of this range. A low inclination is also compatible with the weakness of the emission lines in the spectrum.

It was obvious early in our work that RXJ0944 has a repetitive brightness modulation with a period $\sim 2000 \mathrm{~s}$. With further observations it could be seen that the feature is a double humped profile, with the two humps varying independently and rapidly in amplitude. In Figure 2 we show the light curve of run S6324 on a larger scale, with the cyclic modulation marked, and its highly variable pair of peaks. The FT for this run discloses a fundamental period at $\sim 2220$ s plus its first harmonic. There are only six cycles of this modulation in the light curve, so the uncertainty of the period is large (at least $\sim 40$ $\mathrm{s})$. The mean light curve, folded on the fundamental period of $2162 \mathrm{~s}$ 


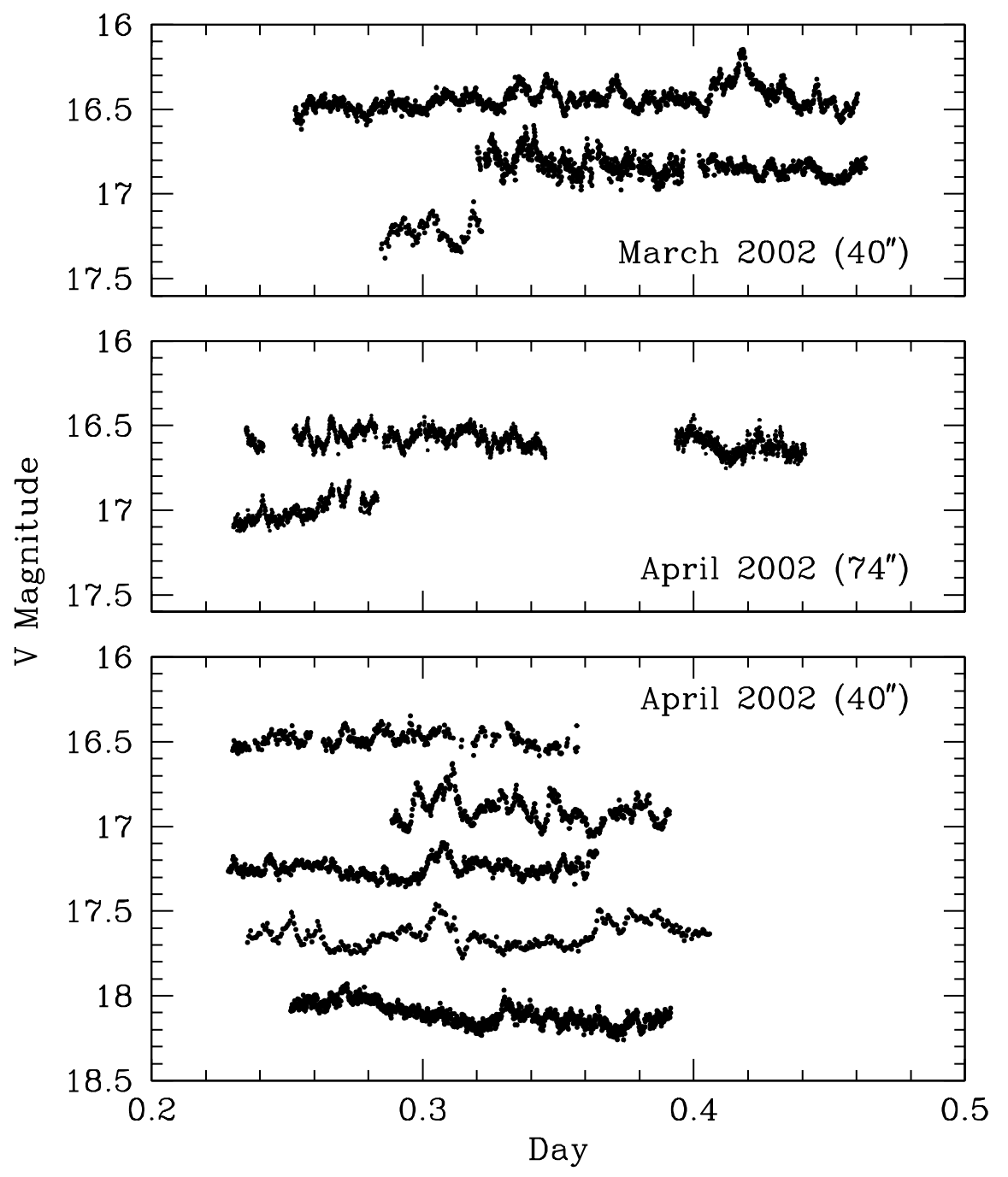

Figure 1. The light curves of RXJ0944, shown in chronological order from top to bottom. In the top panel, runs S6331 and S6340 are displaced downwards by 0.35 and $0.7 \mathrm{mag}$, respectively. In the middle panel run $\mathrm{S} 6350$ is displaced downwards by 0.5 mag. In the lower panel runs S6366, S6370, S6386 and S6391 are displaced vertically by $0.6,0.75,1.1$ and $1.5 \mathrm{mag}$, respectively.

as derived below, is given in Figure 3 and shows the double humped nature of the profile, and that the humps sit on plateaux with only short-lived dips between them. (We removed the strong flare seen at HJD 2452356.418 in Figure 2 as being not representative; it probably resulted from a sudden short-lived surge of mass transference.) In the 


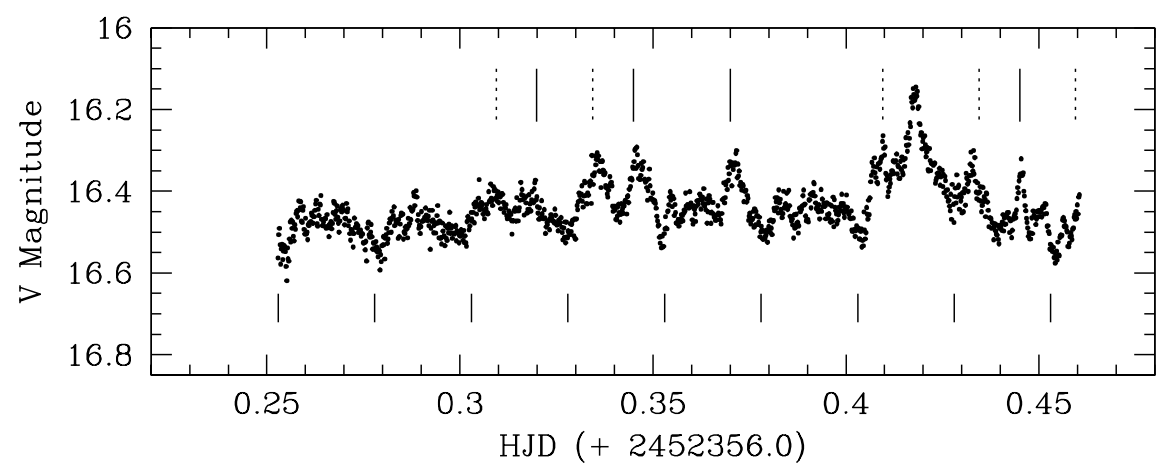

Figure 2. The light curve of RXJ0944 (run S6324). The 2162 s periodicity is marked by the lower vertical bars; the pairs of peaks of variable amplitude are marked by the upper bars (dotted bar for the first peak, solid bar for the second peak).

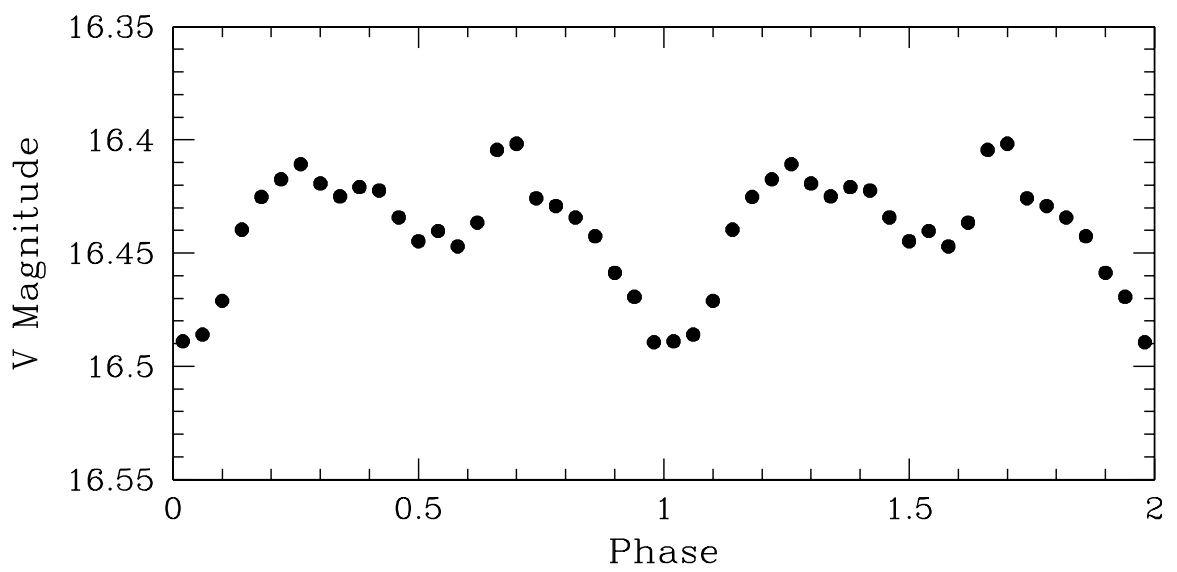

Figure 3. The mean light curve of RXJ0944 (run S6324), folded on the $2162 \mathrm{~s}$ periodicity.

mean light curve, the two peaks occur at about phases 0.26 and 0.68 , respectively.

The peaks on the plateau appear as flares of variable width, so that adding more observations tends to even out their contributions, with the result that the mean light curve for the entire data set (using the period of $2162 \mathrm{~s}$ ), shown in Figure 4, has largely lost the evidence for the doubling of the profile. The FT for the full set of observations is given in Figure 5, and shows clearly the humps of power near the $\sim 2000 \mathrm{~s}$ fundamental and its first and second harmonics. There is a great deal of complicated fine structure in the FT, beyond what is produced by the window pattern; this is caused by the rapid amplitude modulation of the fundamental and its harmonics. It is not possible to 


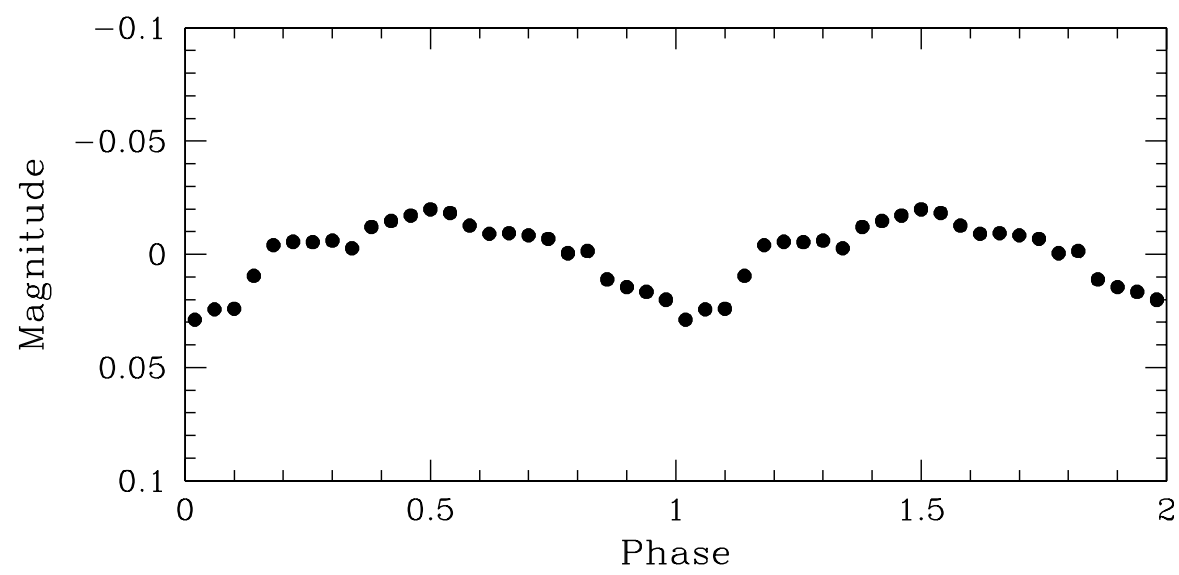

Figure 4. The mean light curve of RXJ0944 (all observations), folded on the $2162 \mathrm{~s}$ periodicity.

select unambiguous frequencies from the forest of aliases. However, the highest peak in the neighbourhood of the fundamental modulation is at $2162 \mathrm{~s}$ and the highest peak at the first harmonic is $1079 \mathrm{~s}$, which supports the choice of a fundamental period near $2160 \mathrm{~s}$.

There are other humps of power in the total FT, but by subdividing our data (in particular, treating the March and April data sets separately) we find that the FT is non-stationary - only the $2160 \mathrm{~s}$ modulation and its harmonics are persistent features. Given the high activity in the light curves (Figure 1) it is not surprising that the FT is also very variable.

We find no evidence for rapid oscillations in brightness (Dwarf Nova Oscillations - typically with periods in the range 5-50 s: see Warner 1995), but in run S6341 we find a Quasi-Periodic Oscillation (QPO; see Warner 1995) with a mean period of $351 \mathrm{~s}$ and amplitude 0.013 mag. This is clearly seen in the light curve and maintains coherence for about 6 cycles between each major change of phase.

\section{Discussion}

The presence of two distinct coherent periodicities in a CV is the recognised signature of an intermediate polar (IP) in which the non-orbital modulation is the spin period $\left(P_{s p}\right)$ of the white dwarf primary, or its orbital side band (see, e.g., Warner 1995). X-ray emission is another common feature of IPs, resulting from accretion from the inner edge of the accretion disc onto the magnetic pole(s) of the white dwarf. We 


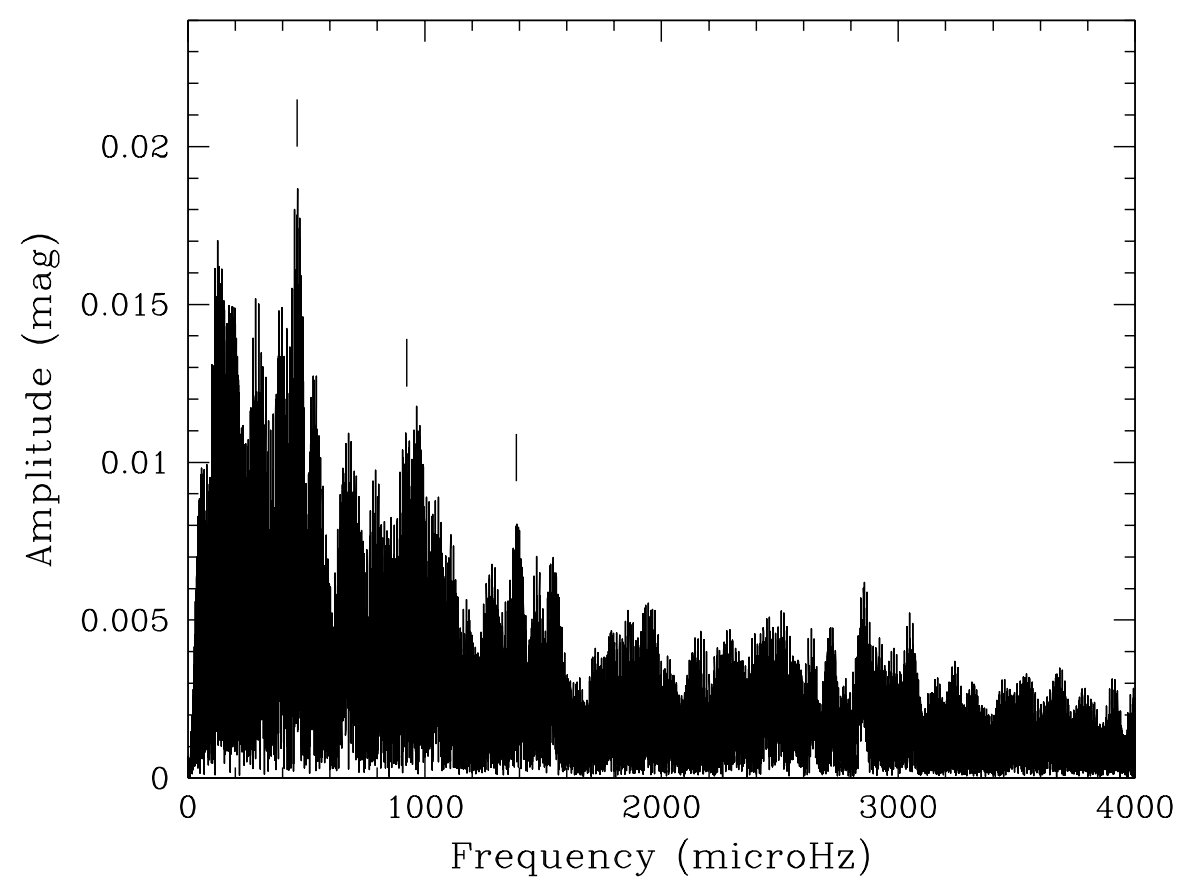

Figure 5. The Fourier Transform of the complete data set of RXJ0944. The fundamental and the first two harmonics of the $2162 \mathrm{~s}$ periodicity are marked by vertical bars.

therefore conclude that RXJ0944 is most probably an IP with highly variable two-pole accretion.

With $P_{\text {orb }}=3.581 \mathrm{~h}$ and $P_{s p}=36.0 \mathrm{~min}$, RXJ0944 is quantitatively similar to canonical IPs such as FO Aqr and TV Col. However, the double-humped light curve and other properties make it most similar to YY Dra, as can be seen from the following brief review of the latter's properties.

YY Dra is a dwarf nova at $\mathrm{V} \sim 16.0$ quiescent magnitude with a mean outburst interval of $870 \mathrm{~d}$ and amplitude $5.5 \mathrm{mag}$, a $P_{\text {orb }}$ of $3.96 \mathrm{~h}$ and a $P_{s p}$ of $529.2 \mathrm{~s}$. Both the spin period and the orbital sideband (at $550 \mathrm{~s}$ ) have been detected in the optical region (Patterson et al. 1992). YY Dra is the X-Ray source 3A1148+719 and the spin modulation is seen in the X-Ray emission (Patterson \& Szkody 1993). An M-type spectrum of the secondary is visible in the red, which is not normal for CVs with $P_{\text {orb }} \sim 4$ h, suggesting a lower luminosity disc, probably the result if its central truncation by the magnetosphere of the primary.

HST observations of YY Dra (Haswell et al. 1997) show that the UV emission line profiles are modulated at half the spin period, and that there is simultaneous presence of broad red and blue wings in C IV 
emission, which is interpreted as evidence for two-pole accretion. This is in accord with the double-humped pulse profiles in the optical and $\mathrm{X}$-ray regions, and the occasional variations in height of the two peaks (though YY Dra is also notable for the near equality of its accretion pole luminosities most of the time). During an outburst of YY Dra X-ray emission greatly increased and the 529 s oscillation was usually visible, but near maximum disappeared, which is interpreted as possible due to the equality of accretion onto two extended poles (Szkody et al. 2002).

There are other IPs with evidence of two-pole accretion: V405 Aur (Haberl et al. 1994) is similar in having its principal optical modulation at the first harmonic rather than the $545 \mathrm{~s}$ fundamental (Allan et al. 1996); the 1WGA J1958.2+3232 ( $\left.P_{s p}=1467 \mathrm{~s}\right)$ has a double peaked profile which shows reversal of circular polarization between the peaks, confirming that it is a two-pole accretor (Norton et al. 2002) and Still, Duck \& Marsh (1998) have found spectroscopic evidence that RX J0558+5353 is a two-pole accretor.

The basic similarity of the optical photometric properties of RXJ0944 and YY Dra is evident, so we conclude that the same model, namely two-pole accretion, is the most probable description of RXJ0944, though with more variable and independent accretion rates onto the two poles. The phases of the two peaks determined from the mean light curve (Figure 3) are not half a cycle apart, indicating that the magnetic poles are not diametrically opposite on the surface of the primary. For an IP, however, the strengths of the He II and C III/N III emission lines at $4686 \AA$ and $4650 \AA$ (Mennickent et al. 2002) are relatively weak but the fact that they are seen at all is uncharacteristic of an ordinary dwarf nova.

\section{Conclusion}

RXJ0944 has the spectroscopic and photometric characteristics of an IP and would be worth studying in a pointed X-ray observation, in order to detect any modulation and thereby determine whether the $36.0 \mathrm{~min}$ periodicity in the optical is the white dwarf spin period or an orbital sideband. A time-resolved spectroscopic study with the HST should also be undertaken. RXJ0944 also has interest as a dwarf nova - there are other intermediate polars that show full dwarf nova outbursts (XY Ari is an example) and others that have abbreviated outbursts (e.g. V1223 Sgr). High speed photometry during an outburst of RXJ0944 could help to reveal the interaction between disc and magnetosphere as the rate of mass transfer increases and decreases. 


\section{Acknowledgements}

We thank Drs S. Potter and P. Rodriguez-Gil for allowing us to use their light curve of RXJ0944. PAW is supported by funds from the University of Cape Town and the National Research Foundation; BW is supported by funds from the University.

\section{References}

Allan, A., Horne, K., Hellier, C., Mukai, K., Barwig, H., Bennie, P. J. and Hilditch, R.W.: 1996, Mon. Not. R. astr. Soc. 279, 1345.

Haberl, F., Thorstensen, J.R., Motch, C., Schwarzenberg-Czerny, A., Pakull, M., Shambrook, A. and Pietsch, W.: 1994, Astron. Astrophys. 291, 171.

Hagen H.-J., Groote, D., Engels, R. and Reimers, D.: 1995, Astron. Astrophys. Suppl. $111,195$.

Haswell, C. A., Patterson, J., Thorstensen, J. R., Hellier, C. and Skillman, D. R.: 1997, Astrophys. J. 476, 847.

Jiang, X. J., Engels, D., Wei, J. Y., Tesch, F. and Hu, J. Y.: 2000, Astron. Astrophys. $362,263$.

Mennickent, R. E., Tovmassian, G., Zharikov, S. V., Tappert, C., Greiner, J., Gaensicke, B. T. and Fried, R. E.: 2002, Astron. Astrophys. 383, 933.

Norton, A. J., Quaintrell, H., Katajainen, S., Lehto, H. J., Mukai, K. and Negueruela, I.: 2002, Astron. Astrophys. 384, 195.

O'Donoghue, D.: 1995, Baltic Astr. 4, 517.

Patterson, J. and Szkody, P.: 1993, Publ. Astron. Soc. Pacific 105, 1116.

Patterson, J., Schwartz, D. A., Pye, J. P., Blair, W. P., Williams, G. A. and Caillault, J.-P.: 1992, Astrophys. J. 392, 233.

Still, M. D., Duck., S. R. and Marsh, T. R.: 1998, Mon. Not. R. astr. Soc. 299, 759.

Szkody, P., Nishikida, K., Erb, D., Mukai, K., Hellier, C., Uemura, M., Kato, T., Pavlenko, E., Katysheva, N., Shugarov, S. and Cook, L.: 2002, Astron. J. 123, 413.

Warner, B.: 1995, Cataclysmic Variable Stars, Cambridge Univ. Press, Cambridge. 